\title{
Virtual Standardized Patients for Assessing the Competencies of Psychologists
}

\author{
Thomas D. Parsons \\ University of North Texas, USA
}

\section{INTRODUCTION}

The development of clinical competence involves having the requisite knowledge and abilities to accomplish clinical tasks. Training of clinicians aims to progress the student from novice to at least the initial level of a competent clinician. Each skill has a developmental trajectory (Spruill et al., 2004). A challenging task is that of developing effective methods for assessing the training interview skills and differential diagnoses. One area of assessment has focused on multiple-choice question examinations. While such tests have been shown to have reliable results, multiple-choice question examinations are often poorly written and tend to favor recall-based knowledge, lack content validity, and include cueing effect of the options (Frederiksen, 1984). Another approach is to make use of essay-based examination, to measure clinical judgment. An obvious limitation of essay questions is the large amount of time involved and the potential for subjective interpretation. Perhaps the most widely used approach over the past couple decades has been the Objective Structured Clinical Examination (OSCE; Doig et al., 2000). The OSCE offers educators an objective and structured competency assessment (Harden, 1988). A limitation of the OSCE is that it involves multiple stations (Barrows, 1993). Current therapeutic training systems that employ the OSCE resort to using real persons (hired actors or students) acting as standardized patients to portray patients with given mental health problems. Training of psychiatrists, for example, has involved the use of standardized patients for decades. In the 1960s, Barrows and Abrahamson (1964) argued for the use of a "programmed" patient (standardized patient) for appraising student performance in the development of clinical skills. In the past decade, the American Association of Medical Colleges has increasingly endorsed the use of standardized patients in teaching and evaluating students' clinical skills (Yudkowsky,
2002). Further, the Accreditation Council for Graduate Medical Education and the National Board of Medical Examiners have encouraged training programs to incorporate standardized patients into assessments of clinical skill development.

\section{BACKGROUND}

Traditional approaches to training clinicians in the interpersonal communication skills needed for assessment, diagnosis, and interview performance rely upon a combination of classroom learning and role-playing with human standardized patients. The importance of interpersonal communication is reflected in recent requirements for communication evaluation in medical schools. The Accreditation Council for Graduate Medical Education (ACGME, 2007) has emphasized the importance of interpersonal and communication skills in training clinicians. Residents are expected to: (1) create and sustain a therapeutic and ethically sound relationship with the patient; (2) use effective listening skills, eliciting and providing information using effective nonverbal, explanatory, questioning, and writing skills; and (3) work in an efficient manner with others. However, evaluation studies have revealed methodological deficiencies in many cases (Chant et al., 2002) and limited positive training effects (Hulsman et al., 1999). In an effort to increase interpersonal communication assessment, standardized patients (paid human actors) have been recruited and trained to exhibit the characteristics of an actual patient, thereby affording novice clinicians a realistic opportunity to practice and to be evaluated in a mock clinical environment. Although a valuable training approach, there are limitations with the use of human standardized patients. For example, human standardized patients are expensive and cost several thousand dollars per student. Further, given the fact that there are

DOI: 10.4018/978-1-4666-5888-2.ch637 
only a handful of sites (for over 130 medical schools in the U.S.) providing standardized patient assessments of the clinician in training's communication ability as part of the U.S. Medical Licensing Examination (USMLE), the current model provides limited availability. Whilst training programs commonly make use of standardized patients to teach interview skills, the diversity of the scenarios standardized patients can characterize is limited by availability of human actors. Further, there is the economic concern related to the time and money needed to train standardized patients. The inclusion of a standardized patient in an OSCE tends to cost approximately $\$ 10$ per hour and then there is the additional $\$ 34$ per student for involvement in an OSCE Cusimano et al. (1994).

Another concern is the issue of standardization. Despite the expense of standardized patient programs, the standardized patients themselves are typically unskilled actors. As a result of common turnover, administrators face considerable challenges for offering psychometrically reliable and valid interactions with the training clinicians. A related issue is the limited scope that the actors are able to portray. As a result, there tends to be an inadequate array of developmentally, socially, and culturally appropriate scenarios. For example, when a clinician has a pediatric focus and needs access to children, it is difficult for the clinician to pretend that the actor is a child. Finally, many clinical cases (e.g., traumatic brain injury) have associated physical symptoms and behaviors (e.g., dilated pupils, spasms, and uncoordinated movements) that simply cannot be accurately portrayed by human actors. Perhaps most damaging is the "standardization" of standardized patients - will they in fact consistently proffer psychometrically reliable and valid interactions with the training clinicians. OSCE-based checklists may not offer a psychometrically valid measurement of clinical competencies because clinical reasoning is understood as pattern recognition (Regehr, Freeman, Robb, Missiha, \& Heisey, 1999).

The plan of this chapter will be as follows: First, in Section 1 "From Virtual Humans to Virtual Standardized Patients," the author will describe past work in virtual humans and the ways in which the virtual human work has been used to develop virtual standardized patients. Next, in Section 2 "From Cognitive Appraisal to Affect Sensitive Virtual Standardized Patients," there will be a discussion of the need to move beyond the outmoded appraisal theories found in much virtual human work to an approach that more closely approximates affective and cognitive modeling found in psychophysiology and the human neurosciences. In Section 3 "Distributed end-user approach" an answer to the problem of time and resource limitations on current approaches to developing affect sensitive virtual standardized patients.

\section{From Virtual Humans to Virtual Standardized Patients}

One proposed answer to some of the difficulties inherent in training persons with standardized patients, hence human actors, is to use virtual humans as patients. Virtual humans ( $\mathrm{VH})$ are developing into powerful interfaces that can enable greatly increased intuitive human like interactions. These virtual human systems consist of characters that have realistic appearances, can think and act like humans, and can express themselves both verbally and non-verbally. Additionally, these virtual humans can listen and understand natural language and see or track limited user interactions with speech or vision systems. Advances in simulated virtual humans afford the possibility of virtual standardized patients that reduce cost, ensure standardization and faithfully model psychiatric symptoms.

Virtual humans are artificially intelligent (AI) agents that control computer generated bodies and can interact with users through speech and gesture in virtual environments (Gratch and Rickel, 2002). Advanced virtual humans are able to engage in rich conversations (Traum et al., 2008), recognize nonverbal cues (Morency et al., 2008), analyze social and emotional factors (Gratch \& Marsella, 2004) and synthesize human communication and nonverbal expressions (Thiebaux et al., 2008). Building virtual humans requires fundamental advances in $\mathrm{AI}$, speech recognition, natural language understanding and generation, dialog management, cognitive modeling and reasoning, virtual human architectures and computer graphics and animations. All of these technologies need to be integrated together into a single system that can work in unison, be expandable, flexible and plug-and-play with different components.

Virtual Human Agent (VHA) technology has evolved to a point where researchers may begin developing mental health applications that make use of virtual reality patients (Dickerson et al., 2005; Johnsen 
et al., 2006; McGee et al., 1998; Stevens et al., 2006; Triolat al., 2006). As underlying enabling technologies continue to evolve and allow us to design functional and usable "structural" clinical virtual environments (VE), the next important challenge will involve "populating" these environments with virtual representations of humans. Over the last five years, the technology for creating virtual humans ( $\mathrm{VHs}$ ) has evolved to the point where they are no longer regarded as simple background characters, but rather can serve a functional interactional role (Gratch et al., 2002; Swartout et al., 2006). This will be vital to create mental health training tools that leverage the use of $\mathrm{VHs}$ for applications that require human-to-human interaction and communication.

Building on the VHtechnology, virtual standardized patients (VSPs) fulfill the role of standardized patients by simulating a particular clinical presentation with a high degree of consistency and realism (Stevens et al., 2005). There is a growing field of research that applies VSPs to training and assessment of bioethics, basic patient communication, interactive conversations, history taking, and clinical assessments (Bickmore \& Giorgino, 2006; Bickmore et al., 2007; Lok et al., 2006; Parsons et al., 2008). Results suggest that VSPs can provide valid and reliable representations of live patients (Kenny et al., 2007; Triola et al., 2006; Andrew et al., 2007). Additionally VSPs enable a precise presentation and control of dynamic perceptual stimuli; along with conversational dialog and interactions, they have the potential to provide ecologically valid assessments that combine the veridical control and rigor of laboratory measures approaching a verisimilitude that reflects real life situations (Parsons et al., 2008; Andrew et al., 2007).Prototypes have been developed for mental health assessment (Kenny et al., 2007; Kenny et al., 2008a; Kenny et al., 2008b; Kenny et al., 2009; Parsons et al., 2009a; Kenny and Parsons, 2011) and extended the behavioral fidelity necessary to support such diagnoses (e.g., physical appearance, gestures and facial expressions, reactions to pain, sweating, autonomic arousal; see Parsons, 2011a).

\section{From Cognitive Appraisal to Affect Sensitive Virtual Standardized Patients}

Much of the current virtual standardized patient work is built on the same architectures that are commonly used in intelligent virtual human research. These virtual human architectures tend to use appraisal models that specify how events, agents and objects are used to elicit an emotional response depending on a set of parameters (e.g., goals, standards and attitudes) representing the subject. In principle, it is possible to model appraisal processes using classical symbolic AI techniques (Picard, 1997; Chwelos \& Oatley, 1994). For example, Gratch (2000; see also Marsella \& Gratch, 2003) are computer scientists and experts in virtual human research. Their approach to appraisal is based on a computational model of Lazarus's (1991) appraisal model, in which the agent environment is represented through a causal-interpretive data structure that emphasizes causal reasoning and the interpretative character of the appraisal process. For this approach, appraisal is understood as a set of feature detectors that map features of the "causal interpretation" onto appraisal variables. According to Gratch and colleagues the interpretation represents the virtual human's current mental view of what events occurred recently, the current value of state predicates, and future plans and goals. Further, the "desirability" of a state is equated with the state's expected utility.

An unfortunate limitation is that these VH models have limited generalizability to the clinical and affective domains found in VSP research. This is because the cognitive and affective models of virtual humans do not generally account for neurophysiological data (Fellous, Armony, \& LeDoux, 2003). Further, as Magnenat- Thalmann and Thalmann (2005) have pointed out in their review of virtual human research, virtual human models of emotional responses tend to be generated from a cognitive point of view and do not adequately take into account the psychophysiological response. While psychologists tend to agree with the contention of computer scientists that appraisal does play a role in many current theories of emotion, most contemporary psychologists studying emotion emphasize the importance of psychophysiological arousal and that emotions are to be understood as cognitive appraisals and are accompanied by autonomic nervous system activity. Although many appraisal models used by computer scientists developing virtual humans contend that cognitive processes (e.g., sensory perception) present verification for the preeminence of appraisal in emotion, contemporary psychologists and neuroscientists indicate that appraisal processes occur following perception and represent a separate 
cognitive process (Izard, 1993). Of course, while most psychologists would agree with computer scientists developing virtual humans that perception is a necessary part of any sensory experience, it is not known whether perceptual processes are the foundation of cognitive models of emotion or if these emotions are concerned with higher order cognitive appraisals that assign meaning and valence (Eckhardt, Norlander, \& Deffenbacher, 2004).

A major limitation of many of the appraisal models developed by computer scientists for virtual human research is that they follow outdated appraisal models that assert specific patterns of physiological changes that may be observed in affect occurrence after the subjective experience of affect. Unfortunately, the cognition first models developed by computer scientists for virtual humans are not supported by psychophysiological research (Cox \& Harrison, 2008). In fact, a common frustration to attempts at developing an adequate scientific approach to emotion has been focus upon constructing theories of the subjective appraisals. Again studies of the neural basis of emotion and emotional learning have instead focused on how the brain detects and evaluates emotional stimuli and how, on the basis of such evaluations, emotional responses are produced (Magnenat-Thalmann \& Thalmann, 2005).

A preferred approach to developing cognitive and affect sensitive virtual human models would include psychophysiological inputs from humans users to the virtual humans during interactions. These additional inputs may be developed into affect-sensitive VSP interfaces that go beyond conventional virtual human models designed by pure (i.e., devoid of psychophysiological metrics) cognitive appraisal principles. An example paradigm for such affect-sensitive VSP interfaces would be similar to the brain-based-devices (BBDs) that are being designed based on biological principles and are programmed to alter their behavior to the environment through self-learning (Edelman, 2006) — as in work being done to develop intelligent robots. A series of devices with sensors and computersimulated brains have been built in Gerald Edelman's (2006) Neurosciences Institute in La Jolla. The robot brains are modeled on human anatomy, complete with versions of visual cortex, inferotemporal cortex, and hippocampus. It is important to note that these biologically based robot brains are not pre-programmed, but evolve neuronal connections in response to experience. These brain-based devices can learn to recognize pat- terns and navigate novel environments. Although the development of such computational models for virtual humans would be difficult, researchers (MagnenatThalmann \& Thalmann, 2005) have pointed out that computational approaches to emotional processing are both possible and practical (see Parsons, 2011a). Nevertheless, the preparation of a virtual human to conduct even a relatively basic free-form conversation can take computer science experts months. The move from a basic appraisal model to a psychophysiologically enhanced and affect sensitive virtual standardized patients would require years of collaborative work between computer scientists and psychologists. When the focus is developing virtual standardized patients for clinical competencies, a revised approach is needed - an approach that can move beyond the labor-intensive and time restrictive requirements found in much virtual human research.

\section{Distributed End-User Approach}

Much of virtual human (see Gratch, 2000; Marsella \& Gratch, 2003) and virtual standardized patient work has involved computer scientists and psychologists (Parsons et al., 2008; Kenny et al., 2007; Kenny et al., 2008a; Kenny et al., 2008b; Kenny et al., 2009; Parsons et al., 2009a; Kenny \& Parsons, 2011) working together as the hub for transferring information from experts and novices to the conversation corpus. Rosen, Lind, and Lok (2009) have shown that such an approach is limited: 1) corpus retrieval necessitates a corpus comprehensive enough for generalization; 2) logistical limitations in the use of the corpora sources relative to legal use of existing material, cost, time, and end-user availability; and 3) computer scientists are the primary developers, but unless they have psychologists that know the domain their virtual humans will fail to represent a virtual standardized patient. A result of these limitations is that there is an increase in response errors and a decreased ability for the clinician's interaction with the virtual standardized patient to achieve competency-based training objectives.

Benjamin Lok's group has moved beyond the limited paradigm discussed above to develop a method that addresses these challenges by directly engaging end-users in the process of knowledge acquisition for conversational modeling. Lok's group has developed an approach in which psychologists using virtual hu- 
mans (as opposed to the computer science developers) generate the clinical model using Human-centered Distributed Conversational Modeling (HDCM). HDCM incorporates concepts and designs from crowdsourcing (Singh et al., 2002) and human-computation (Von Ahn $\&$ Dabbish, 2004) to the enumeration of the questionresponse dialectic found in conversation between a psychologist and client. Hence the knowledge base reflects the conversation of the domain experts (i.e., psychologists).

Lok's group has extended the HDCM approach to a web application called Virtual People Factory (VPF) that implements the HDCM process and a web service that provides support for presentation in multiple display mediums. Within the HDCM approach, the computer scientists developing the virtual standardized patient create a conversational model through the collection of knowledge from the clinicians and then use the psychiatric knowledge to develop the conversational model. Domain experts (i.e. psychologists) and programming experts (i.e. computer scientists) collaborate asynchronously through a graphical user interface that is useable by the clinician without any knowledge of the computer programming details of conversational modeling (e.g. XML).

In summary, this approach allows for interactions between a training clinician and a virtual standardized patient, in which domain novices (i.e. computer programmers) enumerate the space of what will be said to the virtual standardized patient; while domain experts (i.e. psychologists) enumerate the space of what the virtual standardized patient will respond. Comparison of this approach to that of past work in virtual humans and virtual standardized patient reveals that Lok's approach allows for faster completion with a much larger corpus of end-users. As a result, a corpus is generated that enumerates the space of a conversation and forms the basis of a virtual standardized patient model for corpus retrieval used in the assessment of clinical competencies (see Filichia et al., 2010).

\section{FUTURE RESEARCH DIRECTIONS}

There are a number of limitations inherent in the preparation of a virtual humans to conduct even a relatively basic free-form conversation. Current approaches can take computer science experts months to years. Further, the move from a basic appraisal model to a psychophysiologically enhanced and affect sensitive virtual standardized patients would require years of collaborative work between computer scientists and psychologists. When the focus is developing virtual standardized patients for clinical competencies, a revised approach is needed - an approach that can move beyond the labor-intensive and time restrictive requirements found in much virtual human research.

One exciting possibility is the work of Lok and colleagues. In their work they have developed a web application called Virtual People Factory (VPF) that implements the HDCM process and a web service that provides support for presentation in multiple display mediums. This approach allows for interactions between a training clinician and a virtual standardized patient, in which domain novices (i.e. computer programmers) enumerate the space of what will be said to the virtual standardized patient; while domain experts (i.e. psychologists) enumerate the space of what the virtual standardized patient will respond. Comparison of this approach to that of past work in virtual humans and virtual standardized patient reveals that Lok's approach allows for faster completion with a much larger corpus of end-users. As a result, a corpus is generated that enumerates the space of a conversation and forms the basis of a virtual standardized patient model for corpus retrieval used in the assessment of clinical competencies.

The fact that virtual humans and virtual standardized patients can now be developed by clinicians using web-based graphical user interfaces is a stimulating advancement. Technological advances in computing and the World Wide Web in the last couple decades

(Abbate, 1999) have allowed for Internet-based virtual worlds, in which psychologists can interact with potentially more diverse samples in respect to socioeconomic status, sex, and age than traditional samples that are often drawn from undergraduate students (Gosling et al., 2004). Virtual worlds are made up of online communities in which persons interrelate in simulated environments. The continued progress in the development of robust technologies such as more rapid and secure Internet connections has led to the ever increasing interest in social networks (Boulos \& Wheeler, 2007). Virtual worlds provide users to experience social interaction as they participate in individual and group activities. The Virtual People Factory is a web-based application that implements the 
HDCM process and a web service that provides support implementation in virtual worlds. The combination of virtual world technology, virtual standardized patients, and clinical training protocols offers exciting potential for advancing the assessment and training of clinical competencies.

\section{CONCLUSION}

Since virtual humans and virtual environments can allow for precise presentation and control of dynamic perceptual stimuli, conversations and interactions, they can provide ecologically valid therapeutic assessments and treatment that combine the control and rigor of laboratory measures with a verisimilitude that reflects real life situations (Parsons, 2011b). Research into the use of VSPs in psychotherapy training is in its nascent stages. Although progress has been made toward establishing systems that are sensitive to component psychological processes, more studies are required to understand the effectiveness of these systems for training and education, to measure the believability of the characters with respect to their verbal and nonverbal behavior, and the ways in which differences in gender, ethnicity, and personality impact interactions with the VSPs.

\section{REFERENCES}

Accreditation Council for Graduate Medical Education. (2010). ACGME Outcome Project. Retrieved December 5, 2007, from www.acgme.org/Outcomes

Andrew, R., \& Johnsen, K. (2007). Comparing Interpersonal Interactions with a Virtual Human to those with a Real Human. IEEE Transactions on Visualization and Computer Graphics, 13, 443-457. doi:10.1109/ TVCG.2007.1030 PMID:17356212

Barrows, H. (1993). An overview of the uses of standardized patients for teaching and evaluating clinical skills. Academic Medicine, 68, 443453. doi:10.1097/00001888-199306000-00002 PMID:8507309
Barrows, H. S., \& Abrahamson, S. (1964). The programmed patient: a technique for appraising student performance in clinical neurology. Journal of Medical Education, 39, 802-805. PMID:14180699

Bickmore, T., \& Giorgino, T. (2006). Health Dialog Systems for Patients and Consumers. Journal of Biomedical Informatics, 39, 556-571. doi:10.1016/j. jbi.2005.12.004 PMID:16464643

Bickmore, T., Pfeifer, L., \& Paasche-Orlow, M. (2007). Health Document Explanation by Virtual Agents. Lecture Notes in Computer Science, 4722, 183-196. doi:10.1007/978-3-540-74997-4_18

Chant, S., Jenkinson, T., Randle, J., Russell, G., \& Webb, C. (2002). Communication skills training in healthcare: A review of the literature. Nurse Education Today, 22, 189-202. doi:10.1054/nedt.2001.0690 PMID: 12027600

Chwelos, G., \& Oatley, K. (1994). Appraisal, Computational Models, and Scherer's Expert System. Cognition and Emotion, 8, 245-257. doi:10.1080/02699939408408940

Cox, D. E., \& Harrison, D. W. (2008). Models of anger: contributions from psychophysiology, neuropsychology and the cognitive behavioral perspective. Brain Structure \& Function, 212, 371-385. doi:10.1007/ s00429-007-0168-7 PMID:18197417

Cusimano, M., Cohen, R., \& Tucker, W. (1994). A comparative analysis of the costs of administration of an OSCE. Academic Medicine, 69, 571-576. doi:10.1097/00001888-199407000-00014 PMID:8018269

Dickerson, R., Johnsen, K., Raij, A., Lok, B., Hernandez, J., \& Stevens, A. (2005). Evaluating a scriptbased approach for simulating patient-doctor interaction. In Proceedings of the International Conference of Human-Computer Interface Advances for Modeling and Simulation (pp. 79-84).

Doig, C., Harasym, P., \& Fick, G. (2000). An objective look at OSCE. Academic Medicine, 75(10), 96-98. doi:10.1097/00001888-200010001-00031

Eckhardt, C. I., Norlander, B., \& Deffenbacher, J. (2004). The assessment of anger and hostility: a critical review. Aggression and Violent Behavior, 9, 17-43. doi:10.1016/S1359-1789(02)00116-7 
Edelman, G. M. (2006). Second Nature: Brain Science and Human Knowledge. New Haven, CT: Yale University Press.

Fellous, J.-M., Armony, J. L., \& LeDoux, J. E. (2003). Emotional Circuits and Computational Neuroscience. In The Handbook of Brain Theory and Neural Networks (pp. 398-401). Cambridge, MA: The MIT Press.

Filichia, L., Halan, S., Tech, B., Blackwelder, E., Rossen, B., \& Lok, B. et al. (2010). Description of Web-Enhanced Virtual Character Simulation System to Standardize Patient Hand-Offs. The Journal of Surgical Research, 1-6. PMID:20828726

Frederiksen, N. (1984). The real test bias. The American Psychologist, 39, 193-202. doi:10.1037/0003066X.39.3.193

Gratch, J. (2000). Émile: marshaling passions in training and education. In Proceedings of the Fourth International Conferenced on Intelligent Agents. Barcelona, Spain.

Gratch, J., \& Marsella, S. (2004). A domain independent framework for modeling emotion. Journal of Cognitive Systems Research, 5, 269-306. doi:10.1016/j. cogsys.2004.02.002

Gratch, J., \& Rickel, J. (2002). Creating Interactive Virtual Humans: Some Assembly Required.IEEE Intelligent Systems, 54-61. doi:10.1109/MIS.2002.1024753

Harden, R. M.(1988). Whatis an OSCE? MedicalTeacher, 10(1), 19-22. doi:10.3109/01421598809019321 PMID:3221760

Hulsman, R. L., Gos, W. J. G., Winnubst, J. A. M., \& Bensing, J. M. (1999). Teaching clinically experienced physicians communication skills: A review of evaluation studies. Medical Education, 33, 655-668. doi:10.1046/j.1365-2923.1999.00519.x PMID:10476016

Izard, C. E. (1993). Organizational and motivational functions of discrete emotions. In M. Lewis, \& J. M. Haviland (Eds.), Handbook of emotions. New York: Guilford Press.

Johnsen, K., Dickerson, R., Raij, A., Harrison, C., Lok, B., \& Stevens, A. (2006). Evolving an immersive medical communication skills trainer. Presence (Cambridge, Mass.), 15, 33-46. doi:10.1162/pres.2006.15.1.33
Kenny, P., Parsons, T. D., Gratch, J., Leuski, A., \& Rizzo, A. A. (2007). Virtual Patients for Clinical Therapist Skills Training. Lecture Notes in Artificial Intelligence, 4722, 197-210.

Kenny, P., Parsons, T. D., Gratch, J., \& Rizzo, A. A. (2008b). Evaluation of Justina: A Virtual Patient with PTSD. Lecture Notes in Artificial Intelligence, 5208, 394-408.

Kenny, P., Parsons, T. D., Pataki, C. S., Pato, M., St-George, C., Sugar, J., \& Rizzo, A. A. (2008a). Virtual Justina: A PTSD Virtual Patient for Clinical Classroom Training. Annual Review of Cybertherapy and Telemedicine, 6, 113-118.

Kenny, P., Parsons, T. D., \& Rizzo, A. A. (2009). Human Computer Interaction in Virtual Standardized Patient Systems. Lecture Notes in Computer Science, 5613, 514-523. doi:10.1007/978-3-642-02583-9_56

Lazarus, R. (1991). Emotion and Adaptation. NY: Oxford University Press.

Lok, B., Ferdig, R., Raij, A., Johnsen, K., Dickerson, R., \& Coutts, J. (2006). Applying Virtual Reality in Medical Communication Education: Current Findings and Potential Teaching and Learning Benefits of Immersive Virtual Patients. Journal of Virtual Reality, 10, 185-195. doi:10.1007/s10055-006-0037-3

Magnenat-Thalmann, N., Kim, H., Egges, A., \& Garchery, S. (2005). Believability and Interaction in Virtual Worlds. In Proceedings of the 11 th International Multimedia Modelling Conference (pp. 2-9).

Magnenat-Thalmann, N., \& Thalmann, D. (2005). Virtual Humans: Thirty Years Of Research, What Next? The Visual Computer, 21, 1-19. doi:10.1007/ s00371-004-0243-5

Marsella, S., \& Gratch, J. (2003). Modeling coping behaviors in virtual humans: Don't worry, be happy. Paper presented at the Second International Joint Conference on Autonomous Agents and Multi-agent Systems, Melbourne, Australia.

McGee, J. B., Neill, J., Goldman, L., \& Casey, E. (1998). Using multimedia virtual patients to enhance the clinical curriculum for medical students. Medinfo, 9, 732-735. PMID:10384556 
Morency, L.-P., \& de Kok, I. (2008). Context-based Recognition during Human Interactions: Automatic Feature Selection and Encoding Dictionary. In Proceedings of the 10th International Conference on Multimodal Interfaces. Chania, Greece: IEEE.

Parsons, T. D. (2011a). Affect-sensitive Virtual Standardized Patient Interface System. In D. Surry, T. Stefurak, \& R. Gray (Eds.), Technology Integration in Higher Education: Social and Organizational Aspects (pp. 201-221). Hershey: IGI Global.

Parsons, T. D. (2011b). Neuropsychological Assessment using Virtual Environments: Enhanced Assessment Technology for Improved Ecological Validity. In S. Brahnam (Ed.), Advanced Computational Intelligence Paradigms in Healthcare: Virtual Reality in Psychotherapy, Rehabilitation, and Assessment (pp. 271-289). Germany: Springer-Verlag. doi:10.1007/978-3-642-17824-5_13

Parsons, T. D., Cosand, L., Courtney, C., Iyer, A., \& Rizzo, A. A. (2009c). Neurocognitive Workload Assessment using the Virtual Reality Cognitive Performance Assessment Test. Lecture Notes in Artificial Intelligence, 5639, 243-252.

Parsons, T. D., Courtney, C., Cosand, L., Iyer, A., Rizzo, A. A., \& Oie, K. (2009d). Assessment of Psychophysiological Differences of West Point Cadets and Civilian Controls Immersed within a Virtual Environment. Lecture Notes in Artificial Intelligence, 5638, 514-523.

Parsons, T. D., Iyer, A., Cosand, L., Courtney, C., \& Rizzo, A. A. (2009b). Neurocognitive and Psychophysiological Analysis of Human Performance within Virtual Reality Environments. Studies in Health Technology and Informatics, 142, 247-252. PMID:19377160

Parsons, T. D., Kenny, P., Cosand, L., Iyer, A., Courtney, C., \& Rizzo, A. A. (2009a). A Virtual Human Agent for Assessing Bias in Novice Therapists. Studies in Health Technology and Informatics, 142, 253-258. PMID:19377161

Parsons, T. D., Kenny, P., Ntuen, C., Pataki, C. S., Pato, M., \& Rizzo, A. A. (2008). Objective Structured Clinical Interview Training using a Virtual Human Patient. Studies in Health Technology and Informatics, 132, 357-362. PMID:18391321
Picard, R. W. (1997).Affective Computing. Cambridge, MA: M.I.T Press.

Regehr, G., Freeman, R., Robb, A., Missiha, N., \& Heisey, R. (1999). OSCE performance evaluations made by standardized patients: Comparing checklist and global rating scores. Academic Medicine, 74, S135-S137.doi:10.1097/00001888-199910000-00064 PMID:10536618

Rossen, B., Lind, S., \& Lok, B. (2009). Human-Centered Distributed Conversational Modeling: Efficient Modeling of Robust Virtual Human Conversations. Lecture notes in artificial intelligence, 5773, 474-481.

Singh, P. (2002). Open Mind Common Sense: Knowledge Acquisition from the General Public. In On the Move to Meaningful Internet Systems 2002: CoopIS, DOA, and ODBASE (pp. 1223-1237).

Spruill, J., Rozensky, R. H. J., Stigall, T. T., Vasquez, M., Bingham, R. P., \& Olvey, C. (2004). Becoming a competent clinician: Basic Competencies in Intervention. Journal of Clinical Psychology, 60, 741-754. doi:10.1002/jclp.20011 PMID:15195337

Stevens, A., Hernandex, J., \& Johnsen, K. (2005). The use of virtual patients to teach medical students communication skills. American Journal of Surgery, 191, 806-811. doi:10.1016/j.amjsurg.2006.03.002 PMID:16720154

Swartout, W., Gratch, J., Hill, R., Hovy, E., Marsella, S., Rickel, J., \& Traum, D. (2006). Toward Virtual Humans. AI Magazine, 27, 1-10.

Thiebaux, M., \& Marshall, A. (2008). Smart-Body: Behavior Realization for Embodied Conversational Agents. Paper presented at the International Conference on Autonomous Agents and Multi-Agent Systems, Portugal.

Traum, D., \& Gratch, J. (2008). Multi-party, Multiissue, Multi-strategy Negotiation for Multi-modal Virtual Agents. In Proceedings of the 8th International Conference on Intelligent Virtual Agents. Tokyo, Japan: Springer. 
Triola, M., Feldman, H., Kalet, A. L., Zabar, S., Kachur, E. K., \& Gillespie, C. (2006). A randomized trial of teaching clinical skills using virtual and live standardized patients. Journal of General Internal Medicine, 21,424-429. doi:10.1111/j.1525-1497.2006.00421.x PMID:16704382

von Ahn, L., \& Dabbish, L. (2004). Labeling images with a computer game. In Proceedings of the SIGCHI conference on Human factors in computing systems (pp. 319-326).

Yudkowsky, R. (2002). Should we use standardized patients instead of real patients for high-stakes exams in psychiatry? Academic Psychiatry, 26, 187-192. doi:10.1176/appi.ap.26.3.187 PMID:12824138

\section{ADDITIONAL READING}

Kenny, P., \& Parsons, T. D. (2010). Building Embodied Conversational Virtual Patients. Proceedings of the Interservice/Industry Training, Simulation and Education Conference (I/ITSEC), Orlando Florida, December 1-4, 2010.

Kenny, P., \& Parsons, T. D. (2011). Embodied Conversational Virtual Human Patients. In C. Perez-Marin, $\&$ I. Pascual-Nieto (Eds.), Conversational Agents and Natural Language Interaction: Techniques and Effective Practices (pp. 254-281). Hershey: IGI Global. doi:10.4018/978-1-60960-617-6.ch011

Kenny, P., Parsons, T. D., Gratch, J., \& Rizzo, A. A. (2009). Evaluation of Novice and Expert Interpersonal Interaction Skills with a Virtual Patient. Lecture Notes in Computer Science, 5773,511-512. doi:10.1007/9783-642-04380-2_67

Kenny, P., Parsons, T. D., \& Rizzo, A. A. (2009). A Comparative Analysis between Experts and Novices Interacting with a Virtual Patient with PTSD. Studies in Health Technology and Informatics, 144, 122-124. PMID:19592746

Kenny, P., Parsons, T. D., Rothbaum, B. O., Difede, J., Reger, G., \& Rizzo, A. A. (2009). Optimizing clinical training for the treatment of posttraumatic stress disorderusing virtual patients. Studies in Health Technology and Informatics, 144, 264-268. PMID:19592778
Kenny, P., Parsons, T. D., Rothbaum, B. O., Difede, J. Reger, G., \& Rizzo, A. A. (2009). Optimizing Clinical Training for the Treatment of PTSD using Virtual Patients. Studies in Health Technology and Informatics, 144, 264-268. PMID: 19592778

Macedonio, M., Parsons, T. D., \& Rizzo, A. A. (2007). Immersiveness and Physiological Arousal within Panoramic Video-based Virtual Reality. Cyberpsychology \& Behavior, 10,508-516. doi:10.1089/cpb.2007.9997 PMID: 17711358

Parsons, T. D. (2012) Virtual Simulations and the Second Life Metaverse: Paradigm Shift in Neuropsychological Assessment. In V. Zagalo, T. Morgado. \& A. Boa-Ventura (Eds.), Virtual Worlds, Second Life and Metaverse Platforms: New Communication and Identity Paradigms (pp. 234-250). Hershey: IGIGlobal.

Parsons, T. D. (2014). Virtual Teacher and Classroom for Assessment of Neurodevelopmental Disorders. In S. Brahnam, \& L. C. Jain (Eds.), Serious Games, Alternative Realities, and Play Therapy (pp. 121-137). Germany: Springer-Verlag. doi:10.1007/978-3-64245432-5_7

Pataki, C., Pato, M., Sugar, J., Rizzo, A., St-George, C., Kenny, P., \& Parsons, T. D. (2012). Virtual Patients as a Novel Teaching Tool in Psychiatry. Academic Psychiatry, 36, 398-400. doi:10.1176/appi.ap.10080118 PMID:22983473

\section{KEY TERMS AND DEFINITIONS}

Immersion: State of consciousness where a person experiences being in a virtual environment.

Standardized Patient: Actor trained to act as a real patient in order to simulate a set of symptoms or problems.

Virtual Human: Embodied agent with a graphical front-end that is capable of engaging in conversation with humans.

Virtual Standardized Patients: Virtual humans programmed to portray standardized patient scenarios. 